\title{
A Review of Methods for Bias Correction in Medical Images
}

\author{
Shuang Song, ${ }^{1}$ Yuanjie Zheng, ${ }^{1,2,3,4,5 *}$ and Yunlong $\mathrm{He}^{1 *}$ \\ ${ }^{1}$ School of Information Science and Engineering at Shandong Normal University, Jinan, 250300 China \\ ${ }^{2}$ Key Lab of Intelligent Computing \& Information Security in Universities of Shandong at Shandong Normal University, \\ Jinan, 250300 China \\ ${ }^{3}$ Institute of Biomedical Sciences at Shandong Normal University, Jinan, 250300 China \\ ${ }^{4}$ Shandong Provincial Key Laboratory for Distributed Computer Software Novel Technology at Shandong Normal \\ University, Jinan, 250300 China \\ ${ }^{5}$ Key Lab of Intelligent Information Processing of Shandong Normal University, Jinan, 250300 China
}

\begin{abstract}
Bias field in medical images is an undesirable artifact primarily arises from the improper image acquisition process or the specific properties of the imaged object. This artifact can be characterized by a smooth variation of intensities across the image and significantly degrade many medical image analysis techniques. Studies on bias correction have been investigated extensively over these years. In this paper, we proposed to category and analysis existing bias correction methods, provide a complete review article that enables comparative studies on bias correction in medical images.
\end{abstract}

Keywords: Bias correction, intensity nonuniformity, intensity inhomogeneity, medial images, segmentation

\section{Introduction}

With the rapid evolution of medical imaging systems in recent years, clinical analysis of medical images generated by numerous image acquisition technologies has played an essential role in medical diagnoses. These analysis tasks are needed of precise delineation of abnormal changes caused by human diseases and occur in internal tissues and organs. However, the structural complexity of tissues and organs, or the tremendous numbers of images makes the manual delineation wearisome or even impossible (Valverde et al., 2017; Lladó et al., 2012). As a practicable solution, automated analysis is nowadays generally accepted by medical community and has been widely used for clinical diagnoses (J.H. Lee, Marzelli, Jolesz, \& Yoo, 2009; Gubern-Mérida et al., 2015; Weese \& Lorenz, 2016).

It has been validated that the feasibility of an automated analysis system relies on some basic properties of ideal med-

${ }^{*}$ Corresponding author:

Yuanjie Zheng, School of Information Science and Engineering at Shandong Normal University, Jinan, 250300 China E-mail: zhengyuanjie@gmail.com

Yunlong He, School of Information Science and Engineering at Shandong Normal University, Jinan, 250300 China E-mail: ylhvictor@163.com ical images (Zhang, Brady, \& Smith, 2001; C. Li, Gore, \& Davatzikos, 2014). One of the most widely known is that the images are theoretically piecewise constant with a small number of classes. In other words, the intensity of the same tissue should always be similar in one image and not vary with the location of the tissue. For many automated analysis techniques such as segmentation, classification and registration, this property can facilitate their procedures and make their results reasonable (C. Li, Li, Kao, \& Xu, 2009; C. Li, Xu, Anderson, \& Gore, 2009; Xu, Wan, \& Bian, 2013; C. Li et al., 2014).

However, this ideal condition never happens in reality owing to the presence of an undesirable artifact, which can considerably affect the piecewise constant property in medical images. This artifact is referred to as bias field, shading, intensity inhomogeneity or intensity nonuniformity. It can be obtained by various imaging modalities, such as magnetic resonance imaging (MRI), computer tomography (CT), Xray, optical coherence tomography (OCT) and transmission electron microscopy (TEM), etc, and comes from the imperfections of the image acquisition process. Bias field is usually perceived as a smooth variation of intensities across one image. This effect causes variation in the intensity of the same tissue in the different location within the image, hence invalidates the piecewise constant property of medical images. Although the bias field is difficult to be observed by human eyes, it can greatly degrade the accuracy of many 
automated analysis techniques. Consequently, a number of methods have been proposed to correct for the bias field in medical images.

The methods for bias correction were derived from some earlier literatures (Haselgrove \& Prammer, 1986; McVeigh, Bronskill, \& Henkelman, 1986; Axel, Costantini, \& Listerud, 1987), and later generalized in many studies on the sources of bias field (Simmons, Tofts, Barker, \& Arridge, 1994; Keiper et al., 1998; Liang \& Lauterbur, 2000; Alecci, Collins, Smith, \& Jezzard, 2001). Recently, the correction methods can be classified into two main approaches according to different sources of bias field, namely prospective and retrospective approaches (Vovk, Pernus, \& Likar, 2007; Lefkovits, Lefkovits, \& Vaida, 2015; Pop, Vaida, et al., 2015). Prospective approaches aim at reducing the bias field caused by the imperfect image acquisition process. They include approaches based on adjustments of hardware and acquisition devices, such as phantom-based calibration (Collewet, Davenel, Toussaint, \& Akoka, 2002), multi-coil imaging (Brey \& Narayana, 1988; P. Narayana, Brey, Kulkarni, \& Sievenpiper, 1988), or specific designed medical imaging sequences (Mihara, Iriguchi, \& Ueno, 1998). Such approaches can remove scanner-related bias field, but fail to remove the effect on the acquired image, i.e. the anatomyrelated bias field (Boroomand, Shafiee, Khalvati, Haider, \& Wong, 2015). Retrospective approaches propose to correct for the bias field generated by some specific properties of the imaged object, such as the shape, position, orientation and the specific magnetic permeability of the object. These approaches are based only on image intensities and prior knowledge about imaged objects, and can be further classified into filtering based methods (Lewis \& Fox, 2004; Cohen, DuBois, \& Zeineh, 2000), surface fitting based methods (Dawant, Zijdenbos, \& Margolin, 1993; Brechbühler, Gerig, \& Székely, 1996; Styner, Brechbuhler, Szckely, \& Gerig, 2000; Milles, Zhu, Gimenez, Guttmann, \& Magnin, 2007), segmentation based methods (C. Li et al., 2011, 2014; Xie, Gao, Zhu, \& Zhou, 2015; Banerjee \& Maji, 2015) and histogram based methods (Sled, Zijdenbos, \& Evans, 1998; Mangin, 2000; Milles et al., 2007). Retrospective approaches can deal with both scanner-related and anatomy-related bias field artifacts, thus they are more general and flexible than the prospective approaches.

Several reviews of methods for correction of bias field have been published in the past. Earlier researches in regard to performance comparison of different correction methods have been provided (Velthuizen et al., 1998; Arnold et al., 2001). Afterward, a great variety of strategies related to bias correction were summarized (Belaroussi, Milles, Carme, Zhu, \& Benoit-Cattin, 2006; Hou, 2006). Recently, a complete overview of bias correction methods published until 2007 have been presented (Vovk et al., 2007). In this paper, we propose to categorize and analyze various exist- ing bias correction methods employed to date, and provide a relatively complete discussion contains state-of-the-art techniques in order to enable comparative studies on automatic correction of bias field in medical images.

\section{Models of Bias Field}

The model of bias field in medical images is commonly based upon the assumption that bias field is a low-frequency artifact and perceived as a smooth spatially varying function alters the image intensities (Vovk et al., 2007). The simplest form of this model assumes that bias field is additive or multiplicative. The additive form stems from the superposition of the magnetic field in MRI, while the multiplicative form comes form the sensitivity of the reception coils in MRI (Lefkovits et al., 2015). However, disregard the bias field, the real-world medical images are often corrupted by high-frequency noise (Sled et al., 1998). Therefore, a more appropriate image formation model used to describe the corrupted image $S$ should consider the interaction between bias field $B$, bias-free image $I$ and noise $\eta$.

According to different interactions of $I, B$ and $\eta$, three general formulation models have been proposed for bias correction. The most frequently used model assumes that the noise arises from the imperfection of image acquisition devices (Brechbühler et al., 1996; Styner et al., 2000) and independent of the bias field (Pham \& Prince, 1999; Shattuck, Sandor-Leahy, Schaper, Rottenberg, \& Leahy, 2001). It is a multiplicative model with an additive noise, as expressed below:

$$
S(x, y)=I(x, y) B(x, y)+\eta(x, y)
$$

where $(x, y)$ index pixel in the image, and if an image has $M$ rows and $N$ columns, respectively, we have $1 \leqslant x \leqslant M$ and $1 \leqslant y \leqslant N$. This noise $\eta$ follows a Gaussian probability distribution, namely scanner noise (Sijbers, den Dekker, Scheunders, \& Van Dyck, 1998).

Another formulation model is similar to the first, except that the noise is added before the the bias-free image is corrupted. This model considers that the noise comes from the tissue inhomogeneity and can be scaled by the bias field, and has a higher signal-to-noise ratio (SNR) (Prima, Ayache, Barrick, \& Roberts, 2001; Ashburner \& Friston, 2005). It is defined as follows:

$$
S(x, y)=(I(x, y)+\eta(x, y)) B(x, y)
$$

The third formulation model assumes that the bias field and noise are additive. This model takes the logarithmic transform of intensity to correct for the bias field (Guillemaud \& Brady, 1997; Van Leemput, Maes, Vandermeulen, \& Suetens, 1999; Wells, Grimson, Kikinis, \& Jolesz, 1996), and can be defined as follows:

$$
\log S(x, y)=\log I(x, y)+\log B(x, y)+\eta(x, y)
$$


where the noise $\eta$ is theoretically computed in the logarithmic domain and follows a Gaussian probability distribution. However, in practice, the noise can be easily removed by many simple methods (Vovk et al., 2007). Therefore, in most of bias correction methods, the form of noise term is considered rather irrelevant or not to be considered.

Although there still exist other kinds of models in medical images, many studies have revealed that majority of correction methods are based on the simple and more reliable correction models Eq. (1) - Eq. (3). The author in (Likar, Viergever, \& Pernus, 2001) extended the bias field term $B$ in Eq. (1) into multiplicative and additive components and proposed a new model to correct for the bias field. However, this model is effective in microscopic images (Likar, Maintz, Viergever, Pernus, et al., 2000) but not valid for the popular MR images (Likar et al., 2001). Studies in the literatures (Velthuizen et al., 1998; Sled et al., 1998; Mangin, 2000) have theoretically proved that the model of a smooth multiplicative bias field and additive noise is reasonable. From a hardware perspective, the author in (Collewet et al., 2002) proposed a physics based model, which is different from the simple multiplicative model of bias field. This model is useful in in spin-echo T(1) images, but need some special parameters and references, and the results is still unreliable. Recently, various state-of-the-art techniques sill depend on the three simple image formulation models (Seshamani, Cheng, Fogtmann, Thomason, \& Studholme, 2014; Nascimento, Frery, \& Cintra, 2014; Lui, Modhafar, Glaister, Wong, \& Haider, 2014; Boroomand et al., 2015; Banerjee \& Maji, 2015; Miao et al., 2016; Chang et al., 2016), further validates the general applicability of these three models.

\section{Classification of Correction Methods}

Over the recent two decades, a large numbers of bias correction methods have been proposed. They can be classified into two main approaches: prospective and retrospective. Prospective approaches eliminate the bias field caused by hardware equipment, while retrospective approaches reduce the bias field arises from the properties of imaged object and more general than the prospective approaches. In this section, we will provide a relatively detailed review of both prospective and retrospective approaches.

\section{Prospective Methods}

Prospective methods focus on eliminating the bias field arises from the devices of the image acquisition process, and the bias correction can be done prospectively by calibrating and improving the acquisition process. These methods mainly make use of the images of a uniform phantom, multi-coil imaging, or specific designed medical imaging sequences.
Phantom-Based Calibration. The phantom-based approach for correction of bias field can be accomplished by acquiring an additional image of a uniform phantom with a given physical properties. Several phantom based approaches have been proposed and investigated in the past years (Condon, Patterson, Wyper, Jenkins, \& Hadley, 1987; Tofts et al., 1994; Davenel et al., 1999; Moyher, Vigneron, \& Nelson, 1995; Tincher, Meyer, Gupta, \& Williams, 1993), but none of them have been widely used in practice because of the frequent and time-consuming acquisitions of the phantom images. To deal with that problem, the author in (Wicks, Barker, \& Tofts, 1993) proposed a correction matrix to transform the estimated phantom from just one or two orientations to images of any orientation, and therefore the number of phantom acquisitions can be reduced. Besides, there also exist many studies aim at mathematically modeling the bias field (Tincher et al., 1993; Condon et al., 1987), and then fit the model to the phantom image. The author in (Collewet et al., 2002) provided a physics based mathematical model that effective in improving the results of a standard phantom calibration, but the drawback is too sensitive to the input parameters and references.

Multicoil Imaging. Such approaches for bias correction can be done by acquiring additional images with different coils. The coils can be divided into surface coils and body coils. The surface coils have a high SNR but severe bias field, while the body coils is just the opposite. In order to get a bias-free image with a good SNR, (Brey \& Narayana, 1988) proposed a method to combine the surface and body coil images. The bias field can be acquired by separating the filtered surface coil image and the body coil image, and then smoothing the resulting image (P. Narayana et al., 1988). However, these methods have two main disadvantages. First, they have lengthy image acquisition time. Second, the bias field of body coil image is hard to be completely separated from the surface coil image. In order to shorten acquisition times, The author in (Lai \& Fang, 2003) employed a low resolution body coil image registered to the full resolution surface coil image, then the bias field used for correction can be modeled by a spline surface. Dealing with the incomplete inhomogeneity correction, (Fan et al., 2003) provided a approach to incorporate one body coil with the multiple surface coil images, and then the bias can be obtained for estimation.

Special Sequences. Bias correction can be done by designing special medical imaging sequences. It is because the spatial distribution of the flip angle, which can be mathematically estimated from certain pulse sequences, can be used to calculate the bias field in medical images. The author in (Mihara et al., 1998) employed two flip angles ( $\theta$ and 2 $\theta$ ) to obtain two Spin-Echo images, then the spatial distribution of the flip angle can be computed for bias correction. Furthermore, different from the methods using the same sequence with two different flip angles, the approach proposed 
in the (Thulborn, Boada, Shen, Christensen, \& Reese, 1998) is able to estimate the spatial variation of the flip angle using a specific sequence, for example, the echo-planar imaging (EPI) (Chiou, Ahn, Muftuler, \& Nalcioglu, 2003; Belaroussi et al., 2006). Besides, the methods of sensitivity encoding by multiple receiver coils were mainly developed to speed up the scanning process (Pruessmann, Weiger, Scheidegger, Boesiger, et al., 1999). In general, majority of the special sequences based methods are related to specific acquisition (hardware) designs.

\section{Retrospective}

Retrospective approaches are more general and efficient due to they are based only on the information from acquired images and prior knowledge about imaged objects, but can deal with both scanner- and anatomy-related bias field artifacts. The well-known retrospective approaches for bias correction can be divided into filtering based methods, surface fitting based methods, segmentation based methods and histogram based methods.

Filtering Based Methods. Filtering based methods treat the bias field as a low-frequency artifact that can be removed from the high-frequency components of acquired images by a low-pass filter. These methods are simple and computationally inexpensive and have been widely used in the past years (P. A. Narayana \& Borthakur, 1995; Murakami, Hayes, \& Weinberger, 1996; Johnston, Atkins, Mackiewich, \& Anderson, 1996; Tomaževič, Likar, \& Pernuš, 2002). However, the effectiveness of most conventional methods are quite limited because of two reasons (Brinkmann, Manduca, $\&$ Robb, 1998). First, many useful low-frequency structures might be mistakenly eliminated by low-pass filtering. Second, high contrast structures characterized at the low frequencies may generate filtering artifacts known as edge effects, which can cause distortion of homogeneous tissues near the edges. Later, Luo et al. (Luo, Zhu, Clarysse, \& Magnin, 2005) proposed to use a linear combination of singularity functions to recover low-frequency structures which are lost during filtering. Besides, there also exist many solutions used to handle the edge effects, such as replace background pixels by average intensity values (Johnston et al., 1996; Cohen et al., 2000), or extrapolating tissue intensities (Zhou et al., 2001; Simmons et al., 1994). These methods are more efficient for bias correction. However, substantial bias field still remains in the corrected image after filtering (Arnold et al., 2001). Moreover, robust methods for estimation of the bias field based on wavelet transform (Han, Hatsukami, \& Yuan, 2001; Lin, Chen, Belliveau, \& Wald, 2003) or minimization of the segmentation error (Gispert et al., 2004) have been proved to be effective in estimating the bias field generated by surface coils and phase array coils.

Recently, two main filtering approaches have been widely used for bias correction, namely homomorphic filtering (HF) and homomorphic unsharp masking (HUM) methods, both of them can be performed with a low-pass filter such as mean based (Simmons et al., 1994; Zhou et al., 2001) and median based (P. A. Narayana \& Borthakur, 1995) filters. It has been validated that the mean based filters are more suitable for images of human brain (Brinkmann et al., 1998).

Homomorphic Filtering. $\mathrm{HF}$ is use as a classical bias correction technique conducted on log-transformed image intensities (P. A. Narayana \& Borthakur, 1995; Johnston et al., 1996; Velthuizen et al., 1998; Sreenivasan, Havlicek, \& Deshpande, 2015; Yang, Gach, Li, \& Mutic, 2016). This method able to simultaneously increase contrast and reduce bias field. It extracts the bias field by low-pass filtering (LPF) of the input image, then the corrected image can be obtained by subtracting the bias field from the input image in the logdomain, as expressed below:

$$
\log I(x, y)=\log S(x, y)-\operatorname{LPF}(\log S(x, y))+C_{N}
$$

where the the $\operatorname{LPF}(\cdot)$ is the function of a low-pass filter, and the $C_{N}$ is a normalization constant that used to keep the mean or maximum intensity of the corrected image (Lewis \& Fox, 2004).

Homomorphic Unsharp Masking. HUM was first proposed by Axel (Axel et al., 1987). This method is an approximation of classical HF, but is conducted on the original domain (non-log-transformed), which is conceptually straightforward, fast and can be easily implemented. The model of HUM can be defined as blow:

$$
I(x, y)=S(x, y) / B(x, y)=S(x, y) C_{N} / \operatorname{LPF}(S(x, y))
$$

In many cases, HUM maybe the simplest and one of the most commonly used methods for correction of the bias field in medical images (Ardizzone, Pirrone, \& Gambino, 2005; Santha Kumari, Suresh, Yashwanth, \& Rao, 2015; Ardizzone, Pirrone, Gambino, \& Vitabile, 2014).

Surface Fitting Based Methods. Surface fitting based methods aim to model the bias field as a parametric surface which is usually a polynomial or spline function. In the modeling procedure, the parametric surface is fitted to a set of image features that contain information about bias field. Once a surface is generated, the bias field can be acquired by extrapolating all points from the surface, then the correction is equivalently implemented using Eq. (1) or Eq. (3) (Velthuizen et al., 1998). Regardless of the polynomial or spline function used in the fitting process, the performance of these methods relies heavily on the selection of image features. According to different types of image features used for surface fitting, these methods can be further classified into the intensity based and gradient based approaches.

Intensity Based. Intensity based methods assume that the intensities of one type of tissue do not vary significantly unless they are corrupted by bias field. Therefore, the intensity variation inside a region composed of the same type of 
tissues can be used to estimate the bias field. Dawant et al. (Dawant et al., 1993) proposed a least squares method to fit the thin plate splines to intensities of a set of manually selected or automatically selected points, which are distributed over the entire image but belong to the same tissue. Their experiment indicated that the manual selection can lead to better results, but is time-consuming and subjective. Zhuge et al. (Zhuge, Udupa, Liu, Saha, \& Iwanage, 2002) provided a more efficient automatic approach to iteratively fitting a second order polynomial to intensities of the tissue produced by segmentation of homogeneous areas of the dominant tissue. Moreover, Vemuri et al. (Vemuri, Kholmovski, Parker, \& Chapman, 2005) used a Gaussian dominant tissue model to approximate the surface on every tissue regions, thus the bias field across one entire tissue can be integrally estimated. The main drawback of the intensity based methods is that the intensity of just one major tissue may not contain the bias field across the whole image, especially when the homogeneous areas is too large.

Gradient Based. The gradient based methods assume that the large bias areas are evenly distributed over the entire image and can be corrected for by estimating the local variation of intensity gradients. Meyer et al. (Meyer, Bland, \& Pipe, 1995) propose to fit a polynomial surface to underlying normalized intensities of segmented homogeneous areas obtained from a segmented dataset. In order to minimize the difference between derivatives of bias field and the corresponding surface model, Lai et al. (Lai \& Fang, 1999) proposed a finite element surface to model the bias field and lead to good correction performance. There also exist gradient based methods that can estimate the bias field based on derivative of an integral (Vokurka, Watson, Watson, Thacker, \& Jackson, 2001; Vokurka, Thacker, \& Jackson, 1999). However, the gradient based methods are effective only when the homogeneous areas are very large. For a general image, these methods may introduce other adverse image information (Vovk et al., 2007).

Segmentation Based Method. Segmentation based methods perform alternating bias correction and image segmentation steps. They consider that bias correction is commonly served as a necessary preprocessing step for facilitating image segmentation, and conversely, accurate segmentation makes the bias correction insignificant. Therefore, they merge the segmentation and bias correction steps into a united framework to simultaneously yielding better results of each step. According to different image segmentation method utilized, these bias correction methods can be further classified into approaches based on expectationmaximization (EM) algorithm and fuzzy c-means (FCM) algorithm.

EM Based. In EM based approaches, the expectationmaximization (EM) algorithm are used for interleaved segmentation and bias correction. These methods use paramet- ric models which are based on a given probability criterion to estimate the bias field. The maximum-likelihood (ML) or maximum a posterior (MAP) probability is the frequently used probability criterion. Once the criterion and model have been chosen, the model's parameters need to be estimated by the EM algorithm.

EM algorithm was first used for segmentation of human brain images (Wells et al., 1996). In this method, EM algorithm is used to estimate a finite Gaussian mixture (FGM) model which is based on the MAP criterion in order to jointly perform bias field correction and simultaneous segmentation. Guillemaud and Brady (Guillemaud \& Brady, 1997) purposed to improve the FGM models by introducing an additional tissue class which had a uniform intensity probability distribution to model the intensities not belonging to any of the major tissues. A similar idea is to use an mixed tissue classes to model the partial volume effect $(\mathrm{X} . \mathrm{Li}, \mathrm{Li}, \mathrm{Lu}, \&$ Liang, 2005). Gispert et al. proposed and refined a unique approach to improve the EM iterative scheme by using a new criterion named classification error rate (CER) (Gispert et al., 2003, 2004). Bansal et al. proposed an EM based algorithm by using the criterion of minimum image entropy (Bansal, Staib, \& Peterson, 2004). Moreover, the expectation conditional maximization (ECM) algorithm (Prima et al., 2001; Kim, Ng, McLachlan, \& Wang, 2003), the adaptative generalized EM (AGEM) algorithm (Van Leemput et al., 1999) or the iterative conditional modes (ICM) algorithm (X. Li et al., 2005) extended the conventional EM algorithm for the problems of vast number of searched parameters.

Fuzzy C-Means Based. The fuzzy c-means methods using energy minimization to perform simultaneous segmentation and bias correction in which the standard fuzzy cmeans (FCM) algorithm is used for segmentation. The FCM was originally used for MR images segmentation (James, 1981), not appropriate for bias correction. Lee and Vannier (S. K. Lee \& Vannier, 1996) employed the FCM to deal with the bias field by adapting the clustering to local variations. Pham and Prince (Pham \& Prince, 1999) proposed an extension of FCM by using a bias field as a factor in the cluster centers. This approach can be formulated as an energy minimization scheme based on the fuzzy c-means (FCM) algorithm, called adaptive FCM (AFCM). In order to improve the availability of AFCM, in their later publication, Pham extended AFCM to an improved formulation scheme named FANTASM by adding a spatial regularization on the tissue membership functions (Pham, 2001). Moreover, Ahmed et al. proposed a new method to modify the original FCM. This method adds a constraint term to introduce the neighbor information of the pixels labels (Ahmed, Yamany, Mohamed, Farag, \& Moriarty, 2002). Recent methods based on fuzzy c-means are more popular and attractive. Aparajeeta et al. proposed three modified FCM algorithms to segment the given MR image while estimating the bias field by the 
notions of possibilistic and fuzzy membership (Aparajeeta, Nanda, \& Das, 2016). A new method called Bias Estimated Spatial Fuzzy C-means based on the spatial neighbourhood information have been presented (Adhikari, Sing, \& Basu, 2016). A hierarchical model based on multivariate student t-distribution has been provided (Chen, Zhang, Zheng, Jeon, $\& \mathrm{Wu}, 2016)$.

Histogram Based Method. Histogram based methods exploit the image histogram to automatically correct for the bias field in medical images. Many of these methods require an initialization or a priori knowledge about the intensity or gradient probability distribution of the imaged structures. According to the different prior knowledge used, these methods can be further classified into intensity distribution based and gradient distribution based methods.

Intensity Distribution Based. Intensity distribution has been employed by various bias correction methods. In 1998, a classical method known as the nonparametric nonuniform normalization (N3) was proposed (Sled et al., 1998). This method iteratively seeks the smooth multiplicative field by maximizing the high frequency component of the image intensity distribution. As a defacto standard in this field, N3 is easy to implement and has relatively outstanding performance for a number of imaging subjects (Arnold et al., 2001; Boyes et al., 2008; W. Zheng, Chee, \& Zagorodnov, 2009). Recently, an improved N3 method named N4 for bias correction has been proposed (Tustison et al., 2010). In this method, corrected image at each iteration is computed by using the results of previous iteration, in contrast, $\mathrm{N} 3$ estimates a "total bias field" in each iteration. Their experiment shown that $\mathrm{N} 4$ are more superior in terms of effectiveness and efficiency.

There still exist the method estimates bias field by estimating the intensity distribution such as by image entropy or by histogram matching (Vovk et al., 2007). Viola et al. proposed to consider the image entropy (Viola \& Wells III, 1997), and this method later applied in microscopic (Likar et al., 2000) and MR images (Likar et al., 2000, 2001). An iterative method to correct for the bias field by reducing the global entropy of the feature space has been presented (Vovk, Pernuš, \& Likar, 2004). This method was extended by incoperate the spatial and intensity information from multispectral MR images (Vovk, Pernuš, \& Likar, 2006). Vovk et al. proposed a histogram method to correct for the bias field by fitting a B-spline bias field to local estimates of image nonuniformity, and the local estimates are computed based on the image histogram of local intensity distribution (Shattuck et al., 2001). Styner et al. presented a histogram method strategy by modeling the Legendre polynomial inhomogeneity as a special valley function (Styner et al., 2000). Their experiment has demonstrated the high degree of robustness and versatility of this method.
Gradient Distribution Based. Recently, a method estimates the bias field by using the sparseness property of the gradient probability distribution has been proposed (Y. Zheng, Grossman, Awate, \& Gee, 2009). This method is based on the assumption that the gradient of real-world images obey a sparse probability distribution (Olshausen et al., 1996; Y. Zheng, Yu, Kang, Lin, \& Kambhamettu, 2008), which can be characterized by a high kurtosis and two heavy tails. This sparse distribution model can be expressed as:

$$
\rho(x)=e^{-|x|^{\alpha}}
$$

where the parameter $\alpha<1$ and can be fit from the gradient histogram. In Zheng et al.'s method, the image gradient histogram with the optimal bin size is computed to fit the Eq. (6) with the maximum likelihood (Aldrich et al., 1997; Shimazaki \& Shinomoto, 2007). The method is easy to implement due to it works by solving an iteratively re-weighted least squares (IRLS) problem. The experimental results indicate that this method has outstanding performance on the images object provided by different imaging modalities.

\section{Conclusion}

This paper reviewed various existing methods for bias correction in medical images. These methods were categorized and analysed according to difference sources of bias field or features used in the process of correcting. It is worth attention that although the number of issues corresponding to different methods have been studied for many years, bias field correction is still completely solved problem. The seductive strategies have been investigated constantly, for example, a method based on the sparseness of the gradient distribution (Y. Zheng et al., 2008), which has been used successfully vignetting correction and has recently applied in medical images (Y. Zheng et al., 2009). In the future, we hope that the sparseness property, which can be treated as a robust prior knowledge of an ideal image or an ideal deformation field, may work following many medical analysis techniques such as segmentation, classification, or registration in medical images.

\section{Acknowledgments}

This work was made possible through support from Natural Science Foundation of China (NSFC) (61572300), Natural Science Foundation of Shandong Province in China (ZR2014FM001) and Taishan Scholar Program of Shandong Province in China (TSHW201502038).

\section{References}

Adhikari, S. K., Sing, J. K., \& Basu, D. K. (2016). Bias field estimation and segmentation of mri images using a spatial fuzzy cmeans algorithm. In Control, instrumentation, energy $\mathcal{G}$ communication (ciec), 2016 2nd international conference on (pp. 158162). 
Ahmed, M. N., Yamany, S. M., Mohamed, N., Farag, A. A., \& Moriarty, T. (2002). A modified fuzzy c-means algorithm for bias field estimation and segmentation of mri data. IEEE transactions on medical imaging, 21(3), 193-199.

Aldrich, J., et al. (1997). Ra fisher and the making of maximum likelihood 1912-1922. Statistical Science, 12(3), 162-176.

Alecci, M., Collins, C. M., Smith, M. B., \& Jezzard, P. (2001). Radio frequency magnetic field mapping of a 3 tesla birdcage coil: experimental and theoretical dependence on sample properties. Magnetic resonance in medicine, 46(2), 379-385.

Aparajeeta, J., Nanda, P. K., \& Das, N. (2016). Modified possibilistic fuzzy c-means algorithms for segmentation of magnetic resonance image. Applied Soft Computing, 41, 104-119.

Ardizzone, E., Pirrone, R., \& Gambino, O. (2005). Frequency determined homomorphic unsharp masking algorithm on knee $\mathrm{mr}$ images. In International conference on image analysis and processing (pp. 922-929).

Ardizzone, E., Pirrone, R., Gambino, O., \& Vitabile, S. (2014). Illumination correction on biomedical images. Computing and Informatics, 33(1), 175-196.

Arnold, J. B., Liow, J.-S., Schaper, K. A., Stern, J. J., Sled, J. G., Shattuck, D. W., ... others (2001). Qualitative and quantitative evaluation of six algorithms for correcting intensity nonuniformity effects. NeuroImage, 13(5), 931-943.

Ashburner, J., \& Friston, K. J. (2005). Unified segmentation. Neuroimage, 26(3), 839-851.

Axel, L., Costantini, J., \& Listerud, J. (1987). Intensity correction in surface-coil mr imaging. American Journal of Roentgenology, 148(2), 418-420.

Banerjee, A., \& Maji, P. (2015). Rough sets and stomped normal distribution for simultaneous segmentation and bias field correction in brain $\mathrm{mr}$ images. IEEE Transactions on Image Processing, 24(12), 5764-5776.

Bansal, R., Staib, L. H., \& Peterson, B. S. (2004). Correcting nonuniformities in mri intensities using entropy minimization based on an elastic model. In International conference on medical image computing and computer-assisted intervention (pp. 78-86).

Belaroussi, B., Milles, J., Carme, S., Zhu, Y. M., \& Benoit-Cattin, H. (2006). Intensity non-uniformity correction in mri: existing methods and their validation. Medical Image Analysis, 10(2), 234-246.

Boroomand, A., Shafiee, M. J., Khalvati, F., Haider, M. A., \& Wong, A. (2015). Noise-compensated, bias-corrected diffusion weighted endorectal magnetic resonance imaging via a stochastically fully-connected joint conditional random field model. arXiv preprint arXiv:1512.04636.

Boyes, R. G., Gunter, J. L., Frost, C., Janke, A. L., Yeatman, T., Hill, D. L., ... others (2008). Intensity non-uniformity correction using $\mathrm{n} 3$ on 3 -t scanners with multichannel phased array coils. Neuroimage, 39(4), 1752-1762.

Brechbühler, C., Gerig, G., \& Székely, G. (1996). Compensation of spatial inhomogeneity in mri based on a parametric bias estimate. In Visualization in biomedical computing (pp. 141-146).

Brey, W. W., \& Narayana, P. A. (1988). Correction for intensity falloff in surface coil magnetic resonance imaging. Medical Physics, 15(2), 241-245.

Brinkmann, B. H., Manduca, A., \& Robb, R. A. (1998). Optimized homomorphic unsharp masking for mr grayscale inhomogeneity correction. IEEE transactions on medical imaging, 17(2), 161171.

Chang, H., Huang, W., Wu, C., Huang, S., Guan, C., Sekar, S., ... Duan, Y. (2016). A new variational method for bias correction and its applications to rodent brain extraction. IEEE Transactions on Medical Imaging.

Chen, Y., Zhang, H., Zheng, Y., Jeon, B., \& Wu, Q. J. (2016). An improved anisotropic hierarchical fuzzy c-means method based on multivariate student t-distribution for brain mri segmentation. Pattern Recognition, 60, 778-792.

Chiou, J.-Y., Ahn, C. B., Muftuler, L. T., \& Nalcioglu, O. (2003). A simple simultaneous geometric and intensity correction method for echo-planar imaging by epi-based phase modulation. IEEE transactions on medical imaging, 22(2), 200-205.

Cohen, M. S., DuBois, R. M., \& Zeineh, M. M. (2000). Rapid and effective correction of rf inhomogeneity for high field magnetic resonance imaging. Human brain mapping, 10(4), 204-211.

Collewet, G., Davenel, A., Toussaint, C., \& Akoka, S. (2002). Correction of intensity nonuniformity in spin-echo 1 -weighted images. Magnetic resonance imaging, 20(4), 365-373.

Condon, B., Patterson, J., Wyper, D., Jenkins, A., \& Hadley, D. (1987). Image non-uniformity in magnetic resonance imaging: its magnitude and methods for its correction. The British journal of radiology, 60(709), 83-87.

Davenel, A., Marchal, P., Riaublanc, A., Gandemer, G., Belton, P., Hills, B., \& Webb, G. (1999). Magnetic resonance mapping of solid fat content of adipose tissues in meat. SPECIAL PUBLICATION-ROYAL SOCIETY OF CHEMISTRY, 231, 272279.

Dawant, B. M., Zijdenbos, A. P., \& Margolin, R. A. (1993). Correction of intensity variations in $\mathrm{mr}$ images for computer-aided tissue classification. IEEE transactions on medical imaging, 12(4), 770-781.

Fan, A., Wells, W. M., Fisher, J. W., Cetin, M., Haker, S., Mulkern, R., ... Willsky, A. S. (2003). A unified variational approach to denoising and bias correction in mr. In Biennial international conference on information processing in medical imaging (pp. 148-159).

Gispert, J. D., Reig, S., Pascau, J., Lazaro, M., Vaquero, J. J., \& Desco, M. (2003). Inhomogeneity correction of magnetic resonance images by minimization of intensity overlapping. In Image processing, 2003. icip 2003. proceedings. 2003 international conference on (Vol. 2, pp. II-847).

Gispert, J. D., Reig, S., Pascau, J., Vaquero, J. J., García-Barreno, P., \& Desco, M. (2004). Method for bias field correction of brain t1-weighted magnetic resonance images minimizing segmentation error. Human brain mapping, 22(2), 133-144.

Gubern-Mérida, A., Martí, R., Melendez, J., Hauth, J. L., Mann, R. M., Karssemeijer, N., \& Platel, B. (2015). Automated localization of breast cancer in dce-mri. Medical image analysis, 20(1), 265-274.

Guillemaud, R., \& Brady, M. (1997). Estimating the bias field of $\mathrm{mr}$ images. IEEE Transactions on Medical imaging, 16(3), 238-251.

Han, C., Hatsukami, T. S., \& Yuan, C. (2001). A multi-scale method for automatic correction of intensity non-uniformity in $\mathrm{mr}$ images. Journal of Magnetic Resonance Imaging, 13(3), 
$428-436$.

Haselgrove, J., \& Prammer, M. (1986). An algorithm for compensation of surface-coil images for sensitivity of the surface coil. Magnetic Resonance Imaging, 4(6), 469-472.

Hou, Z. (2006). A review on $\mathrm{mr}$ image intensity inhomogeneity correction. International Journal of Biomedical Imaging, 2006.

James, C. B. (1981). Pattern recognition with fuzzy objective function algorithms. Kluwer Academic Publishers.

Johnston, B., Atkins, M. S., Mackiewich, B., \& Anderson, M. (1996). Segmentation of multiple sclerosis lesions in intensity corrected multispectral mri. IEEE Transactions on Medical Imaging, 15(2), 154-169.

Keiper, M. D., Grossman, R. I., Hirsch, J. A., Bolinger, L., Ott, I. L., Mannon, L. J., ... Kolson, D. L. (1998). Mr identification of white matter abnormalities in multiple sclerosis: a comparison between $1.5 \mathrm{t}$ and $4 \mathrm{t}$. American journal of neuroradiology, 19(8), 1489-1493.

Kim, S.-G., Ng, S.-K., McLachlan, G., \& Wang, D. (2003). Segmentation of brain mr images with bias field correction. In Workshop on digital image computing (pp. 3-8).

Lai, S.-H., \& Fang, M. (1999). A new variational shape-fromorientation approach to correcting intensity inhomogeneities in magnetic resonance images. Medical Image Analysis, 3(4), 409424.

Lai, S.-H., \& Fang, M. (2003). A dual image approach for bias field correction in magnetic resonance imaging. Magnetic resonance imaging, 21(2), 121-125.

Lee, J.-H., Marzelli, M., Jolesz, F. A., \& Yoo, S.-S. (2009). Automated classification of fmri data employing trial-based imagery tasks. Medical image analysis, 13(3), 392-404.

Lee, S. K., \& Vannier, M. W. (1996). Post-acquisition correction of $\mathrm{mr}$ inhomogeneities. Magnetic Resonance in Medicine, 36(2), 275-286.

Lefkovits, L., Lefkovits, S., \& Vaida, M.-F. (2015). An atlas based performance evaluation of inhomogeneity correcting effects. MACRo 2015, 1(1), 79-90.

Lewis, E. B., \& Fox, N. C. (2004). Correction of differential intensity inhomogeneity in longitudinal $\mathrm{mr}$ images. Neuroimage, 23(1), 75-83.

Li, C., Gore, J. C., \& Davatzikos, C. (2014). Multiplicative intrinsic component optimization (mico) for mri bias field estimation and tissue segmentation. Magnetic resonance imaging, 32(7), 913923.

Li, C., Huang, R., Ding, Z., Gatenby, J. C., Metaxas, D. N., \& Gore, J. C. (2011). A level set method for image segmentation in the presence of intensity inhomogeneities with application to mri. IEEE Transactions on Image Processing, 20(7), 2007-2016.

Li, C., Li, F., Kao, C.-Y., \& Xu, C. (2009). Image segmentation with simultaneous illumination and reflectance estimation: An energy minimization approach. In 2009 ieee 12th international conference on computer vision (pp. 702-708).

Li, C., Xu, C., Anderson, A. W., \& Gore, J. C. (2009). Mri tissue classification and bias field estimation based on coherent local intensity clustering: A unified energy minimization framework. In International conference on information processing in medical imaging (pp. 288-299).

Li, X., Li, L., Lu, H., \& Liang, Z. (2005). Partial volume segmentation of brain magnetic resonance images based on maximum a posteriori probability. Medical Physics, 32(7), 2337-2345.

Liang, Z.-P. ., \& Lauterbur, P. C. (2000). Principles of magnetic resonance imaging: a signal processing perspective. ąřTheąś Institute of Electrical and Electronics Engineers Press.

Likar, B., Maintz, J. A., Viergever, M. A., Pernus, F., et al. (2000). Retrospective shading correction based on entropy minimization. Journal of Microscopy, 197(3), 285-295.

Likar, B., Viergever, M. A., \& Pernus, F. (2001). Retrospective correction of $\mathrm{mr}$ intensity inhomogeneity by information minimization. IEEE transactions on medical imaging, 20(12), 13981410.

Lin, F.-H., Chen, Y.-J., Belliveau, J. W., \& Wald, L. L. (2003). A wavelet-based approximation of surface coil sensitivity profiles for correction of image intensity inhomogeneity and parallel imaging reconstruction. Human brain mapping, 19(2), 96111.

Lladó, X., Oliver, A., Cabezas, M., Freixenet, J., Vilanova, J. C., Quiles, A., ... Rovira, À. (2012). Segmentation of multiple sclerosis lesions in brain mri: a review of automated approaches. Information Sciences, 186(1), 164-185.

Lui, D., Modhafar, A., Glaister, J., Wong, A., \& Haider, M. A. (2014). Monte carlo bias field correction in endorectal diffusion imaging. IEEE Transactions on Biomedical Engineering, 61(2), 368-380.

Luo, J., Zhu, Y., Clarysse, P., \& Magnin, I. (2005). Correction of bias field in $\mathrm{mr}$ images using singularity function analysis. IEEE transactions on medical imaging, 24(8), 1067-1085.

Mangin, J.-F. (2000). Entropy minimization for automatic correction of intensity nonuniformity. In Mathematical methods in biomedical image analysis, 2000. proceedings. ieee workshop on (pp. 162-169).

McVeigh, E. R., Bronskill, M., \& Henkelman, R. (1986). Phase and sensitivity of receiver coils in magnetic resonance imaging. Medical physics, 13(6), 806-814.

Meyer, C. R., Bland, P. H., \& Pipe, J. (1995). Retrospective correction of intensity inhomogeneities in mri. IEEE Transactions on Medical Imaging, 14(1), 36-41.

Miao, Y., Dai, J., Li, Y., He, W., Shi, W., He, F., ... Zhang, H. (2016). An mr image segmentation algorithm based on bias field correction. In Natural computation, fuzzy systems and knowledge discovery (icnc-fskd), 2016 12th international conference on (pp. 1858-1862).

Mihara, H., Iriguchi, N., \& Ueno, S. (1998). A method of rf inhomogeneity correction in $\mathrm{mr}$ imaging. Magnetic Resonance Materials in Physics, Biology and Medicine, 7(2), 115-120.

Milles, J., Zhu, Y. M., Gimenez, G., Guttmann, C. R., \& Magnin, I. E. (2007). Mri intensity nonuniformity correction using simultaneously spatial and gray-level histogram information. Computerized Medical Imaging and Graphics, 31(2), 81-90.

Moyher, S. E., Vigneron, D. B., \& Nelson, S. J. (1995). Surface coil $\mathrm{mr}$ imaging of the human brain with an analytic reception profile correction. Journal of Magnetic Resonance Imaging, 5(2), 139144.

Murakami, J. W., Hayes, C. E., \& Weinberger, E. (1996). Intensity correction of phased-array surface coil images. Magnetic Resonance in Medicine, 35(4), 585-590.

Narayana, P., Brey, W., Kulkarni, M., \& Sievenpiper, C. (1988). Compensation for surface coil sensitivity variation in magnetic 
resonance imaging. Magnetic resonance imaging, 6(3), 271274.

Narayana, P. A., \& Borthakur, A. (1995). Effect of radio frequency inhomogeneity correction on the reproducibility of intra-cranial volumes using mr image data. Magnetic Resonance in Medicine, 33(3), 396-400.

Nascimento, A. D., Frery, A. C., \& Cintra, R. J. (2014). Bias correction and modified profile likelihood under the wishart complex distribution. IEEE Transactions on Geoscience and Remote Sensing, 52(8), 4932-4941.

Olshausen, B. A., et al. (1996). Emergence of simple-cell receptive field properties by learning a sparse code for natural images. Nature, 381(6583), 607-609.

Pham, D. L. (2001). Spatial models for fuzzy clustering. Computer vision and image understanding, 84(2), 285-297.

Pham, D. L., \& Prince, J. L. (1999). Adaptive fuzzy segmentation of magnetic resonance images. IEEE transactions on medical imaging, 18(9), 737-752.

Pop, P., Vaida, M.-F., et al. (2015). Bias field inhomogeneity measurements. In E-health and bioengineering conference (ehb), 2015 (pp. 1-4).

Prima, S., Ayache, N., Barrick, T., \& Roberts, N. (2001). Maximum likelihood estimation of the bias field in $\mathrm{mr}$ brain images: Investigating different modelings of the imaging process. In International conference on medical image computing and computerassisted intervention (pp. 811-819).

Pruessmann, K. P., Weiger, M., Scheidegger, M. B., Boesiger, P., et al. (1999). Sense: sensitivity encoding for fast mri. Magnetic resonance in medicine, 42(5), 952-962.

Santha Kumari, T., Suresh, B., Yashwanth, P., \& Rao, R. R. (2015). Modified histogram based contrast enhancement using unsharp masking filter for medical images. IJRCCT, 4(2), 137-140.

Seshamani, S., Cheng, X., Fogtmann, M., Thomason, M. E., \& Studholme, C. (2014). A method for handling intensity inhomogenieties in fmri sequences of moving anatomy of the early developing brain. Medical image analysis, 18(2), 285-300.

Shattuck, D. W., Sandor-Leahy, S. R., Schaper, K. A., Rottenberg, D. A., \& Leahy, R. M. (2001). Magnetic resonance image tissue classification using a partial volume model. NeuroImage, 13(5), 856-876.

Shimazaki, H., \& Shinomoto, S. (2007). A method for selecting the bin size of a time histogram. Neural computation, 19(6), 1503-1527.

Sijbers, J., den Dekker, A. J., Scheunders, P., \& Van Dyck, D. (1998). Maximum-likelihood estimation of rician distribution parameters. IEEE Trans. Med. Imaging, 17(3), 357-361.

Simmons, A., Tofts, P. S., Barker, G. J., \& Arridge, S. R. (1994). Sources of intensity nonuniformity in spin echo images at $1.5 \mathrm{t}$. Magnetic Resonance in Medicine, 32(1), 121-128.

Sled, J. G., Zijdenbos, A. P., \& Evans, A. C. (1998). A nonparametric method for automatic correction of intensity nonuniformity in mri data. IEEE transactions on medical imaging, 17(1), 8797.

Sreenivasan, K. R., Havlicek, M., \& Deshpande, G. (2015). Nonparametric hemodynamic deconvolution of fmri using homomorphic filtering. IEEE transactions on medical imaging, 34(5), 1155-1163.

Styner, M., Brechbuhler, C., Szckely, G., \& Gerig, G. (2000).
Parametric estimate of intensity inhomogeneities applied to mri. IEEE transactions on medical imaging, 19(3), 153-165.

Thulborn, K. R., Boada, F. E., Shen, G. X., Christensen, J. D., \& Reese, T. G. (1998). Correction of b1 inhomogeneities using echo-planar imaging of water. Magnetic resonance in medicine, 39(3), 369-375.

Tincher, M., Meyer, C., Gupta, R., \& Williams, D. (1993). Polynomial modeling and reduction of rf body coil spatial inhomogeneity in mri. IEEE transactions on medical imaging, 12(2), 361-365.

Tofts, P. S., Barker, G. J., Simmons, A., MacManus, D. G., Thorpe, J., Gass, A., \& Miller, D. H. (1994). Correction of nonuniformity in images of the spine and optic nerve from fixed receiveonly surface coils at $1.5 \mathrm{t}$. Journal of computer assisted tomography, 18(6), 997-1003.

Tomaževič, D., Likar, B., \& Pernuš, F. (2002). Comparative evaluation of retrospective shading correction methods. Journal of Microscopy, 208(3), 212-223.

Tustison, N. J., Avants, B. B., Cook, P. A., Zheng, Y., Egan, A., Yushkevich, P. A., \& Gee, J. C. (2010). N4itk: improved n3 bias correction. IEEE transactions on medical imaging, 29(6), 1310-1320.

Valverde, S., Oliver, A., Roura, E., González-Villà, S., Pareto, D., Vilanova, J. C., ... Lladó, X. (2017). Automated tissue segmentation of $\mathrm{mr}$ brain images in the presence of white matter lesions. Medical image analysis, 35, 446-457.

Van Leemput, K., Maes, F., Vandermeulen, D., \& Suetens, P. (1999). Automated model-based bias field correction of $\mathrm{mr}$ images of the brain. IEEE transactions on medical imaging, 18(10), 885-896.

Velthuizen, R. P., Heine, J. J., Cantor, A. B., Lin, H., Fletcher, L. M., $\&$ Clarke, L. P. (1998). Review and evaluation of mri nonuniformity corrections for brain tumor response measurements. Medical physics, 25(9), 1655-1666.

Vemuri, P., Kholmovski, E. G., Parker, D. L., \& Chapman, B. E. (2005). Coil sensitivity estimation for optimal snr reconstruction and intensity inhomogeneity correction in phased array $\mathrm{mr}$ imaging. In Biennial international conference on information processing in medical imaging (pp. 603-614).

Viola, P., \& Wells III, W. M. (1997). Alignment by maximization of mutual information. International journal of computer vision, 24(2), 137-154.

Vokurka, E. A., Thacker, N. A., \& Jackson, A. (1999). A fast model independent method for automatic correction of intensity nonuniformity in mri data. Journal of Magnetic Resonance Imaging, 10(4), 550-562.

Vokurka, E. A., Watson, N. A., Watson, Y., Thacker, N. A., \& Jackson, A. (2001). Improved high resolution $\mathrm{mr}$ imaging for surface coils using automated intensity non-uniformity correction: Feasibility study in the orbit. Journal of Magnetic Resonance Imaging, 14(5), 540-546.

Vovk, U., Pernuš, F., \& Likar, B. (2004). Mri intensity inhomogeneity correction by combining intensity and spatial information. Physics in Medicine and Biology, 49(17), 4119.

Vovk, U., Pernuš, F., \& Likar, B. (2006). Intensity inhomogeneity correction of multispectral $\mathrm{mr}$ images. Neuroimage, 32(1), 5461.

Vovk, U., Pernus, F., \& Likar, B. (2007). A review of methods for 
Biomedical Engineering Review, Vol. 1, Issue 1, 2017

A Review of Methods for Bias Correction in Medical Images

correction of intensity inhomogeneity in mri. IEEE transactions on medical imaging, 26(3), 405-421.

Weese, J., \& Lorenz, C. (2016). Four challenges in medical image analysis from an industrial perspective. Medical Image Analysis, $33,44-49$.

Wells, W. M., Grimson, W. E. L., Kikinis, R., \& Jolesz, F. A. (1996). Adaptive segmentation of mri data. IEEE transactions on medical imaging, 15(4), 429-442.

Wicks, D. A., Barker, G. J., \& Tofts, P. S. (1993). Correction of intensity nonuniformity in $\mathrm{mr}$ images of any orientation. Magnetic Resonance Imaging, 11(2), 183-196.

Xie, M., Gao, J., Zhu, C., \& Zhou, Y. (2015). A modified method for mrf segmentation and bias correction of mr image with intensity inhomogeneity. Medical $\mathcal{F}$ biological engineering $\mathcal{F}$ computing, 53(1), 23-35.

Xu, L., Wan, J. W., \& Bian, T. (2013). A continuous method for reducing interpolation artifacts in mutual information-based rigid image registration. IEEE Transactions on Image Processing, 22(8), 2995-3007.

Yang, D., Gach, H., Li, H., \& Mutic, S. (2016). Tu-h-206-04: An effective homomorphic unsharp mask filtering method to correct intensity inhomogeneity in daily treatment $\mathrm{mr}$ images. Medical Physics, 43(6), 3774-3774.

Zhang, Y., Brady, M., \& Smith, S. (2001). Segmentation of brain $\mathrm{mr}$ images through a hidden markov random field model and the expectation-maximization algorithm. IEEE transactions on medical imaging, 20(1), 45-57.

Zheng, W., Chee, M. W., \& Zagorodnov, V. (2009). Improvement of brain segmentation accuracy by optimizing non-uniformity correction using n3. Neuroimage, 48(1), 73-83.

Zheng, Y., Grossman, M., Awate, S., \& Gee, J. (2009). Automatic correction of intensity nonuniformity from sparseness of gradient distribution in medical images. In Medical image computing and computer-assisted intervention: Miccai... international conference on medical image computing and computer-assisted intervention (Vol. 12, p. 852).

Zheng, Y., Yu, J., Kang, S. B., Lin, S., \& Kambhamettu, C. (2008). Single-image vignetting correction using radial gradient symmetry. In Computer vision and pattern recognition, 2008. cvpr 2008. ieee conference on (pp. 1-8).

Zhou, L., Zhu, Y., Bergot, C., Laval-Jeantet, A.-M., Bousson, V., Laredo, J.-D., \& Laval-Jeantet, M. (2001). A method of radiofrequency inhomogeneity correction for brain tissue segmentation in mri. Computerized Medical Imaging and Graphics, 25(5), 379-389.

Zhuge, Y., Udupa, J. K., Liu, J., Saha, P. K., \& Iwanage, T. (2002). Scale-based method for correcting background intensity variation in acquired images. In Medical imaging 2002 (pp. 11031111). 